\title{
Welcome Message for the Second Instructional Course for Adult Brachial Plexus Injuries
}

\section{David Chwei-Chin Chuang, MD*}

Division of Reconstructive Microsurgery, Department of Plastic and Reconstructive Surgery, Chang-Gung Memorial Hospital; Chang-Gung University, College of Medicine, Taoyuan, Taiwan

Dear colleagues around the world:

My name is David Chuang. I am a plastic surgeon at the LinkouChang Gung Memorial Hospital, specializing in the treatment and reconstruction of peripheral nerve injuries, particularly brachial plexus injuries (BPIs). I am excited to inform you about a valuable and unique learning opportunity. On Nov 13-16 of this year, the Chang Gung hospital will host an instructional course for BPI reconstruction in adults. This is our second such course; the first was held 7 years ago (in 2009) and was highly successful. Indeed, participants requested that we continue to host these courses on an ongoing basis. Since that first course, our team has reconstructed over 2000 adult BPIs, and developed new concepts, strategic planning strategies, and techniques for diagnosis and treatment. This second course on adult BPI will include live surgeries, lectures, discussions, and Q and A sessions. Furthermore, it will feature involvement from 7 international experts, including Drs. Alain Gilbert, Christophe Oberlin, and Zoubir Belkheyar from France; Dr. Shu-Feng Wang from mainland China; Dr. Y Hattorri from Japan; Dr. Somsak Leechanvengvongs from Thailand; and Dr. Tessa Gordon from Canada. A rehabilitation therapist and six faculty doctors from the Chang Gung hospital, including four surgeons, one radiologist, and one neurologist, will also be involved in lectures, discussions, and live surgeries. The meeting will last two and a half days. This course offers participants the opportunity to gain a more comprehensive understanding of adult BPI focusing on th following topics: the latest findings from experimental studies; diagnostic techniques including MRI, electrodiagnosis, and other methods used in clinical examinations; surgical treatment strategies; and postoperative care and management strategies. In offering this course, we are committed to providing you with comprehensive information on cutting edge diagnostic and treatment techniques related to BPI.

I sincerely welcome you to join us for this instructional course on Nov. 13-16, 2017 at Chang-Gung Memorial Hospital. We look forward to seeing you.

David Chwei-Chin Chuang, MD

Honorary Chairman

Honorary Editor-in-Chief, International Microsurgery Journal Pesident, World Society for Reconstructive Microsurgery

\section{*Correspondence: David Chwei-Chin Chuang, MD}

Division of Reconstructive Microsurgery, Department of Plastic and Reconstructive Surgery, Chang-Gung Memorial Hospital; Chang-Gung University, College of Medicine, Taoyuan, Taiwan. E-mail: dearchuang@gmail.com Received: May 30, 2017; Accepted: May 31, 2017; Published: June 4, 2017 International Microsurgery Journal. 2017;1(1):3 DOI: $10.24983 /$ scitemed.imj.2017.00018

Copyright $(\mathbb{C} 2017$ The Author(s). This is an open-access article distributed under the terms of the Creative Commons Attribution 4.0 International License (CC-BY). 